\title{
On the integrable deformations of a system related to the motion of two vortices in an ideal incompressible fluid
}

\author{
Cristian Lăzureanu ${ }^{1, *}$, Ciprian Hedrea ${ }^{1, * *}$, and Camelia Petrişor $^{1, * * *}$ \\ ${ }^{1}$ Department of Mathematics, Politehnica University of Timişoara, Timişoara, România
}

\begin{abstract}
Altering the first integrals of an integrable system integrable deformations of the given system are obtained. These integrable deformations are also integrable systems, and they generalize the initial system. In this paper we give a method to construct integrable deformations of maximally superintegrable Hamiltonian mechanical systems with two degrees of freedom.

An integrable deformation of a maximally superintegrable Hamiltonian mechanical system preserves the number of first integrals, but is not a Hamiltonian mechanical system, generally. We construct integrable deformations of the maximally superintegrable Hamiltonian mechanical system that describes the motion of two vortices in an ideal incompressible fluid, and we show that some of these integrable deformations are Hamiltonian mechanical systems too.
\end{abstract}

\section{Introduction}

Hamiltonian mechanical systems play a key role in classical mechanics, classical field theory or quantum mechanics. The equations of motion of such a system are given by a Hamiltonian function, and a perturbation of this Hamiltonian leads to a new Hamiltonian mechanical system. Another class of integrable systems that model dynamic processes is given by the Hamilton-Poisson systems, and if such a system has sufficiently constants of motion, then these give the equations of motion (see, e.g., [10]). Moreover, in [3], altering the constants of motion of the Euler top, a new integrable system, called integrable deformation of the Euler top, was obtained.

Recently, the above-mentioned idea was used to obtain integrable deformations of some three-dimensional Hamilton-Poisson systems, namely Maxwell-Bloch equations [4], Rikitake system [5], and Kermack-McKendrick system [7]. Furthermore, in [6] the integrable deformations method for a three-dimensional system of differential equations was proposed.

In this paper we give a method to construct integrable deformations of maximally superintegrable Hamiltonian mechanical systems with two degrees of freedom by alteration of their first integrals. We obtain new integrable systems which have the same number of first integrals, but which are not Hamiltonian mechanical systems, generally. Considering a particular Hamiltonian mechanical system, we show that some integrable deformations are Hamiltonian mechanical systems too. More precisely, we give integrable deformations of a

\footnotetext{
*e-mail: cristian.lazureanu@upt.ro

**e-mail: ciprian.hedrea@upt.ro

***e-mail: camelia.petrisor@upt.ro
} 
Hamiltonian mechanical system used to model the motion of two vortices in an ideal incompressible fluid, and we show that under some conditions such an integrable deformation is also a Hamiltonian mechanical system.

\section{Integrable deformations of maximally superintegrable Hamiltonian mechanical systems with two degrees of freedom}

In this section we obtain integrable deformations of maximally superintegrable Hamiltonian mechanical systems with two degrees of freedom. For details about Hamiltonian mechanical systems see, e.g. [8, 9].

First, we recall some notions concerning with Hamiltonian mechanics. Let $\Omega \subseteq \mathbb{R}^{4}$ be an open set. We consider the Hamiltonian mechanical system $(\Omega, \omega, H)$, where

$$
\omega=d p_{1} \wedge d q_{1}+d p_{2} \wedge d q_{2}
$$

and $H \in C^{\infty}(\Omega, \mathbb{R})$ is the Hamiltonian function. The symplectic structure is given by the Poisson bracket on $\mathbb{R}^{4}$, namely

$$
\{f, g\}_{\omega}=\sum_{i=1}^{2}\left(\frac{\partial f}{\partial p_{i}} \cdot \frac{\partial g}{\partial q_{i}}-\frac{\partial f}{\partial q_{i}} \cdot \frac{\partial g}{\partial p_{i}}\right),
$$

for every $f, g \in C^{\infty}\left(\mathbb{R}^{4}, \mathbb{R}\right)$, and the equations of motion are given by

$$
\dot{q}_{i}=\frac{\partial H}{\partial p_{i}}=\left\{H, q_{i}\right\}_{\omega}, \quad \dot{p}_{i}=-\frac{\partial H}{\partial q_{i}}=\left\{H, p_{i}\right\}_{\omega}, \quad i \in\{1,2\} .
$$

A function $C \in C^{\infty}(\Omega, \mathbb{R})$ is a first integral (constant of motion) of system (3) if $\dot{C}=\frac{d C}{d t}=0$, which is equivalent with the condition $\{H, C\}_{\omega}=0$. Obviously, the Hamiltonian $H$ is a first integral. System (3) is integrable in Liouville's sense if it possesses two functionally independent integrals of motion $H, C_{1}$. Furthermore, system (3) is maximally superintegrable if it possesses three functionally independent first integrals $H, C_{1}, C_{2}$.

In the sequel, we consider that system (3) is maximally superintegrable. Following [10], there is a rescaling function $v \in C^{1}(\Omega, \mathbb{R})$ such that system (3) is a Hamilton-Poisson system,

$$
\dot{x}_{k}=\left\{H, x_{k}\right\}_{C_{1}, C_{2}}^{v}, \quad k \in\{1,2,3,4\}
$$

where

$$
x_{1}=q_{1}, x_{2}=q_{2}, x_{3}=p_{1}, x_{4}=p_{2},
$$

and the Poisson bracket on $\Omega$ generated by $C_{1}, C_{2}$ is given by

$$
\{f, g\}_{C_{1}, C_{2}}^{v}:=v \frac{\partial\left(C_{1}, C_{2}, f, g\right)}{\partial\left(x_{1}, x_{2}, x_{3}, x_{4}\right)},
$$

for every $f, g \in C^{\infty}(\Omega, \mathbb{R})$, where $\frac{\partial\left(C_{1}, C_{2}, f, g\right)}{\partial\left(x_{1}, x_{2}, x_{3}, x_{4}\right)}$ is the Jacobian determinant.

In the following we give integrable deformations of system (4).

Let $\alpha, \beta, \gamma \in C^{1}(\Omega, \mathbb{R})$ be arbitrary functions such that the functions

$$
I_{1}=C_{1}+g_{1} \alpha, I_{2}=C_{2}+g_{2} \beta, \tilde{H}=H+g_{3} \gamma
$$


are functionally independent on $\Omega$, where $g_{1}, g_{2}, g_{3} \in \mathbb{R}$ are deformation parameters. The functions $I_{1}, I_{2}, \tilde{H}$ give rise to the following Hamilton-Poisson system

$$
\dot{x}_{k}=\left\{\tilde{H}, x_{k}\right\}_{I_{1}, I_{2}}^{v}, k \in\{1, \ldots, 4\},
$$

where the Poisson bracket $\{., .,\}_{I_{1}, I_{2}}^{v}$ follows from (6). Taking into account the properties of determinants, system ( 8 ) becomes

$$
\dot{x}_{k}=\left\{H, x_{k}\right\}_{C_{1}, C_{2}}^{v}+g_{1}\left\{H, x_{k}\right\}_{\alpha, C_{2}}^{v}+g_{2}\left\{H, x_{k}\right\}_{I_{1}, \beta}^{v}+g_{3}\left\{\gamma, x_{k}\right\}_{I_{1}, I_{2}}^{v}, \quad k \in\{1, \ldots, 4\} .
$$

It is easy to see that if $g_{1}=g_{2}=g_{3}=0$, then system (8) is the initial system (4). Consequently, system (9) is a deformation of system (4). Moreover, the functions $I_{1}, I_{2}, \tilde{H}$ are first integrals of system (8), hence (9) is an integrable deformation, which is also a maximally superintegrable system.

\section{Integrable deformations of a system related to the motion of two vortices in an ideal incompressible fluid}

In this section we give integrable deformations of the Hamiltonian mechanical system that describes the motion of two vortices in an ideal incompressible fluid. We also consider a particular integrable deformation that preserves two of the first integrals and that is a Hamiltonian mechanical system under some conditions.

In [2], following the physical description of the problem of $n$ point vortices [1], it is mentioned that the planar motion of two vortices of coordinates $\left(q_{1}, p_{1}\right),\left(q_{2}, p_{2}\right)$ in an ideal incompressible fluid can be modeled by the Hamiltonian

$$
H\left(q_{1}, q_{2}, p_{1}, p_{2}\right)=\ln \left[\left(q_{1}-q_{2}\right)^{2}+\left(p_{1}-p_{2}\right)^{2}\right] .
$$

Therefore, the motion of two vortices in an ideal incompressible fluid has a symplectic realization given by the Hamiltonian mechanical system $(\Omega, \omega, H)$, where $\Omega$ is an open set in $\mathbb{R}^{4}$ which does not contain any point $\left(q_{1}, q_{1}, p_{1}, p_{1}\right)$, and $\omega=d p_{1} \wedge d q_{1}+d p_{2} \wedge d q_{2}$. Using (3), one gets the equations of motion

$$
\begin{aligned}
& \dot{q}_{1}=\frac{2\left(p_{1}-p_{2}\right)}{\left(q_{1}-q_{2}\right)^{2}+\left(p_{1}-p_{2}\right)^{2}}, \quad \dot{q}_{2}=\frac{2\left(p_{2}-p_{1}\right)}{\left(q_{1}-q_{2}\right)^{2}+\left(p_{1}-p_{2}\right)^{2}}, \\
& \dot{p}_{1}=\frac{2\left(q_{2}-q_{1}\right)}{\left(q_{1}-q_{2}\right)^{2}+\left(p_{1}-p_{2}\right)^{2}}, \quad \dot{p}_{2}=\frac{2\left(q_{1}-q_{2}\right)}{\left(q_{1}-q_{2}\right)^{2}+\left(p_{1}-p_{2}\right)^{2}} .
\end{aligned}
$$

In [2], a Nambu-Hamilton realization of this system is given. We notice that it is in fact a Hamilton-Poisson realization. More precisely, recalling that the functions

$$
C_{1}=\frac{1}{2}\left(q_{1}+q_{2}\right), C_{2}=p_{1}+p_{2}
$$

are first integrals of system (11), we obtain that the rescaling function is $v=1$, therefore system (11) can be put in the form (4), namely

$$
\dot{q}_{1}=\left\{H, q_{1}\right\}_{C_{1}, C_{2}}, \dot{q}_{2}=\left\{H, q_{2}\right\}_{C_{1}, C_{2}}, \dot{p}_{1}=\left\{H, p_{1}\right\}_{C_{1}, C_{2}}, \dot{p}_{2}=\left\{H, p_{2}\right\}_{C_{1}, C_{2}},
$$

where the Poisson bracket generated by $C_{1}, C_{2}$ is given by

$$
\{f, g\}_{C_{1}, C_{2}}=\frac{\partial\left(C_{1}, C_{2}, f, g\right)}{\partial\left(q_{1}, q_{2}, p_{1}, p_{2}\right)}=\frac{1}{2} \sum_{i=1}^{2} \sum_{j=1}^{2}(-1)^{i+j}\left(\frac{\partial f}{\partial p_{i}} \cdot \frac{\partial g}{\partial q_{j}}-\frac{\partial f}{\partial q_{i}} \cdot \frac{\partial g}{\partial p_{j}}\right),
$$


for every $f, g \in C^{\infty}(\Omega, \mathbb{R})$.

Now, we can give integrable deformations of system (11) such that the functions $I_{1}, I_{2}$, and $\tilde{H}$ given by (7) are their first integrals. Using (9), we obtain the following

Proposition 1 Let $\Omega$ be an open set in $\mathbb{R}^{4}$ which does not contain any point $\left(q_{1}, q_{1}, p_{1}, p_{1}\right)$ and let $\alpha, \beta, \gamma \in C^{1}(\Omega, \mathbb{R})$ be such that the functions $I_{1}=C_{1}+g_{1} \alpha, I_{2}=C_{2}+g_{2} \beta, \tilde{H}=H+g_{3} \gamma$ are functionally independent on $\Omega$, where $g_{1}, g_{2}, g_{3} \in \mathbb{R}, C_{1}, C_{2}$ are given by (12) and $H$ by (10). Then an integrable deformation of system (11) is given by the following system

$$
\begin{aligned}
& \dot{q}_{1}=\frac{2\left(p_{1}-p_{2}\right)}{\left(q_{1}-q_{2}\right)^{2}+\left(p_{1}-p_{2}\right)^{2}}+g_{1}\left\{H, q_{1}\right\}_{\alpha, C_{2}}+g_{2}\left\{H, q_{1}\right\}_{I_{1}, \beta}+g_{3}\left\{\gamma, q_{1}\right\}_{I_{1}, I_{2}}, \\
& \dot{q}_{2}=\frac{2\left(p_{2}-p_{1}\right)}{\left(q_{1}-q_{2}\right)^{2}+\left(p_{1}-p_{2}\right)^{2}}+g_{1}\left\{H, q_{2}\right\}_{\alpha, C_{2}}+g_{2}\left\{H, q_{2}\right\}_{I_{1}, \beta}+g_{3}\left\{\gamma, q_{2}\right\}_{I_{1}, I_{2}}, \\
& \dot{p}_{1}=\frac{2\left(q_{2}-q_{1}\right)}{\left(q_{1}-q_{2}\right)^{2}+\left(p_{1}-p_{2}\right)^{2}}+g_{1}\left\{H, p_{1}\right\}_{\alpha, C_{2}}+g_{2}\left\{H, p_{1}\right\}_{I_{1}, \beta}+g_{3}\left\{\gamma, p_{1}\right\}_{I_{1}, I_{2}}, \\
& \dot{p}_{2}=\frac{2\left(q_{1}-q_{2}\right)}{\left(q_{1}-q_{2}\right)^{2}+\left(p_{1}-p_{2}\right)^{2}}+g_{1}\left\{H, p_{2}\right\}_{\alpha, C_{2}}+g_{2}\left\{H, p_{2}\right\}_{I_{1}, \beta}+g_{3}\left\{\gamma, p_{2}\right\}_{I_{1}, I_{2}},
\end{aligned}
$$

where the Poisson bracket is given by $\{f, g\}_{u, v}=\frac{\partial(u, v, f, g)}{\partial\left(q_{1}, q_{2}, p_{1}, p_{2}\right)}$.

In order to give a symplectic realization of an integrable deformation (15), we consider those integrable deformations of system (11) that preserve the first integrals $C_{1}$ and $C_{2}$, i.e. $g_{1}=g_{2}=0$, functions $\alpha$ and $\beta$ vanish, and $\tilde{H}=H+g \gamma$. The above system takes the form

$$
\begin{aligned}
& \dot{q}_{1}=\frac{2\left(p_{1}-p_{2}\right)}{\left(q_{1}-q_{2}\right)^{2}+\left(p_{1}-p_{2}\right)^{2}}+g\left\{\gamma, q_{1}\right\}_{C_{1}, C_{2}}, \quad \dot{q}_{2}=\frac{2\left(p_{2}-p_{1}\right)}{\left(q_{1}-q_{2}\right)^{2}+\left(p_{1}-p_{2}\right)^{2}}+g\left\{\gamma, q_{2}\right\}_{C_{1}, C_{2}}, \\
& \dot{p}_{1}=\frac{2\left(q_{2}-q_{1}\right)}{\left(q_{1}-q_{2}\right)^{2}+\left(p_{1}-p_{2}\right)^{2}}+g\left\{\gamma, p_{1}\right\}_{C_{1}, C_{2}}, \quad \dot{p}_{2}=\frac{2\left(q_{1}-q_{2}\right)}{\left(q_{1}-q_{2}\right)^{2}+\left(p_{1}-p_{2}\right)^{2}}+g\left\{\gamma, p_{2}\right\}_{C_{1}, C_{2}},
\end{aligned}
$$

or equivalent,

$$
\begin{aligned}
& \dot{q}_{1}=\frac{2\left(p_{1}-p_{2}\right)}{\left(q_{1}-q_{2}\right)^{2}+\left(p_{1}-p_{2}\right)^{2}}+\frac{g}{2}\left(\frac{\partial \gamma}{\partial p_{1}}-\frac{\partial \gamma}{\partial p_{2}}\right), \\
& \dot{q}_{2}=\frac{2\left(p_{2}-p_{1}\right)}{\left(q_{1}-q_{2}\right)^{2}+\left(p_{1}-p_{2}\right)^{2}}-\frac{g}{2}\left(\frac{\partial \gamma}{\partial p_{1}}-\frac{\partial \gamma}{\partial p_{2}}\right), \\
& \dot{p}_{1}=\frac{2\left(q_{2}-q_{1}\right)}{\left(q_{1}-q_{2}\right)^{2}+\left(p_{1}-p_{2}\right)^{2}}-\frac{g}{2}\left(\frac{\partial \gamma}{\partial q_{1}}-\frac{\partial \gamma}{\partial q_{2}}\right), \\
& \dot{p}_{2}=\frac{2\left(q_{1}-q_{2}\right)}{\left(q_{1}-q_{2}\right)^{2}+\left(p_{1}-p_{2}\right)^{2}}+\frac{g}{2}\left(\frac{\partial \gamma}{\partial q_{1}}-\frac{\partial \gamma}{\partial q_{2}}\right) .
\end{aligned}
$$

On the other hand, the equations of motion of the Hamiltonian mechanical system $(\Omega, \omega, \tilde{H})$ are given by

$$
\begin{aligned}
& \dot{q}_{1}=\frac{2\left(p_{1}-p_{2}\right)}{\left(q_{1}-q_{2}\right)^{2}+\left(p_{1}-p_{2}\right)^{2}}+g \frac{\partial \gamma}{\partial p_{1}}, \quad \dot{q}_{2}=\frac{2\left(p_{2}-p_{1}\right)}{\left(q_{1}-q_{2}\right)^{2}+\left(p_{1}-p_{2}\right)^{2}}+g \frac{\partial \gamma}{\partial p_{2}}, \\
& \dot{p}_{1}=\frac{2\left(q_{2}-q_{1}\right)}{\left(q_{1}-q_{2}\right)^{2}+\left(p_{1}-p_{2}\right)^{2}}-g \frac{\partial \gamma}{\partial q_{1}}, \quad \dot{p}_{2}=\frac{2\left(q_{1}-q_{2}\right)}{\left(q_{1}-q_{2}\right)^{2}+\left(p_{1}-p_{2}\right)^{2}}-g \frac{\partial \gamma}{\partial q_{2}} .
\end{aligned}
$$

Note that the functions $C_{1}$ and $C_{2}$ are not first integrals for system (17). 
Remark 1 Generally, systems (16) and (17) are not identically, hence an integrable deformation of a Hamiltonian mechanical system $(\Omega, \omega, H)$, which preserves the first integrals $C_{1}, C_{2}$ and is obtained by perturbation $\tilde{H}=H+g \gamma$, is not the Hamiltonian mechanical system $(\Omega, \omega, \tilde{H})$.

Imposing the condition that systems (16) and (17) to be identically, we get

$$
\frac{\partial \gamma}{\partial p_{1}}+\frac{\partial \gamma}{\partial p_{2}}=0, \frac{\partial \gamma}{\partial q_{1}}+\frac{\partial \gamma}{\partial q_{2}}=0
$$

Consequently, we have the following result.

Proposition 2 Let $\gamma\left(q_{1}, q_{2}, p_{1}, p_{2}\right)=F\left(q_{1}-q_{2}, p_{1}-p_{2}\right)$, where $F \in C^{1}\left(\mathbb{R}^{2}, \mathbb{R}\right)$ is an arbitrary function. Then the integrable deformation (16) of the Hamiltonian mechanical system $(\Omega, \omega, H)$, where $H$ is given by $(10)$, is a Hamiltonian mechanical system $(\Omega, \omega, \tilde{H})$, where $\tilde{H}=H+g \gamma, g \in \mathbb{R}$.

\section{Conclusions}

In this paper, a method to construct integrable deformations of maximally superintegrable Hamiltonian mechanical systems with two degrees of freedom was presented. This method is used to obtain integrable deformations of the Hamiltonian mechanical system that describes the motion of two vortices in an ideal incompressible fluid. These integrable deformations are also maximally superintegrable systems, but generally they are not Hamiltonian mechanical systems. Conditions as integrable deformations of the system that describes the motion of two vortices in an ideal incompressible fluid to be Hamiltonian mechanical systems are obtained.

\section{Acknowledgment}

This work was supported by research grants PCD-TC-2017.

\section{References}

[1] V. I. Arnold, Dynamical Systems 3 ( Springer-Verlag, Berlin, 1988) 24-25.

[2] R. Chatterjee, Dynamical Symmetries and Nambu Mechanics, Lett. Math. Phys. 36, 117126 (1996).

[3] A. Galajinsky, Remark on integrable deformations of the Euler top, J. Math. Anal. Appl. 416, 995-997 (2014).

[4] C. Lăzureanu, On the Hamilton-Poisson realizations of the integrable deformations of the Maxwell-Bloch equations, C .R. Math. 355 (5), 596-600 (2017).

[5] C. Lăzureanu, Hamilton-Poisson Realizations of the Integrable Deformations of the Rikitake System, Adv. Math. Phys. 2017, Article ID 4596951, 9 pages (2017).

[6] C. Lăzureanu, Integrable Deformations of Three-Dimensional Chaotic Systems, Int. J. Bifurcat. Chaos 28 (5), Article ID 1850066, 7 pages (2018).

[7] C. Lăzureanu and C. Petrişor, Stability and Energy-Casimir Mapping for Integrable Deformations of the Kermack-McKendrick System, Adv. Math. Phys. 2018, Article ID 5398768, 9 pages (2018).

[8] P. Libermann, C.-M. Marle, Symplectic Geometry and Analytical Mechanics (D. Reidel, Dordrecht, 1987).

[9] M. Puta, Hamiltonian Mechanical System and Geometric Quantization (Kluwer Academic Publishers, Dordrecht, Boston, London, 1993).

[10] R.M. Tudoran, A normal form of completely integrable systems, J. Geom. Phys. 62, no. 5, 1167-1174 (2012). 